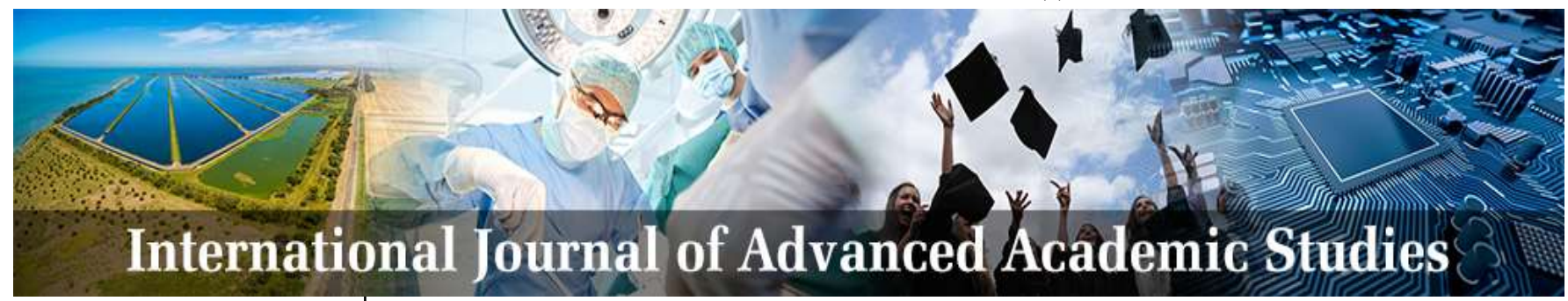

E-ISSN: 2706-8927

P-ISSN: 2706-8919

www.allstudyjournal.com

IJAAS 2021; 3(1): 01-08

Received: 23-10-2020

Accepted: 02-12-2020

\section{Anissa Gara}

a) Institut national

agronomique de Tunisie,

Avenue Charles Nicolle,

Tunisia

b) Laboratory of rural

Economy at Institut national

de recherches agronomiques de

Tunisia

\section{Dorra Ajabi}

Institut national agronomique

de Tunisie, Avenue Charles

Nicolle, Tunisia
Corresponding Author:

Anissa Gara

a) Institut national agronomique de Tunisie,

Avenue Charles Nicolle,

Tunisia

b) Laboratory of rural

Economy at Institut national

de recherches agronomiques de

Tunisia

\section{Relationship between farmlands' size and agricultural practices}

\section{Anissa Gara and Dorra Ajabi}

DOI: https://doi.org/10.33545/27068919.2021.v3.i1a.456

\begin{abstract}
In the field of agriculture, the scientific community has followed studies on methods and tools to meet the growing need to reassess agricultural operations and therefore to judge the degree of deterioration and pollution of natural capital. In this study, the IDEA method -which is Farm Sustainability assessment method-, was used to meet this objective, at Mornag area in Tunisia. This detailed analysis based on the factor of farm size, allowed to identify the characteristics and the strategies for agricultural sustainability of the different groups of farms according to size and upon three scales: agroecological, socio-territorial and economical scales. The results showed that the overall scales' scores at each group are positively affected by farm's size. Indeed, the detailed analyzes of the scores of indicators and components showed that the land size turned out to be a limiting factor against sustainability.
\end{abstract}

Keywords: Farms, size, sustainable agriculture, Tunisia

\section{Introductions}

Harwood (1990), in Zahm et al, (2015) [5], argues that attempting to formulate a definition of sustainable agriculture applicable on a large scale is illusory. In the end, he defines sustainable agriculture as "an agriculture which evolves indefinitely towards greater human utility, a more efficient use of resources, while respecting a balance with the environment, which is favorable to humans as well as to other species". Sustainable agriculture is therefore an application of the concept of sustainable development to the agricultural sector and more specifically at the farm level. It takes into account the three dimensions: economic, social and environmental in a global framework. In addition, a farm is, by extension, sustainable if it generates enough income to support the family, if it implements environmentally friendly practices, if it contributes to the social integration of farms and if it is transmissible (Grolleau, 2001) ${ }^{[1]}$. Since the 1990s Tunisia has been engaged in a process of sustainable development by establishing strategies aimed at sustainable agriculture. This perspective was essential given that agricultural sector in Tunisia encloses several failures at the environmental, social, and economic levels, which threatens the sustainability of farms.

In addition, land tenure situation of agricultural land in Tunisia is characterized by remarkable fragmentation, which often prevents the application of good agricultural practices, especially in terms of resource conservation and the use of good agricultural equipment. The smaller the farm, the more difficult it is to invest in efficient and economical agricultural machinery and equipment. Moreover, Tunisian lands are threatened by human factors, namely urban sprawl and constructions and therefore the loss of fertile agricultural land. The losses of agricultural land following urbanization are estimated at between 2000 and 3000 hectares per year, despite the presence of legislation in force which controls or prohibits this practice (Massin et al, 2016) ${ }^{[2]}$. On the other hand, small producers - owning small-sized farmlands - are facing problems in financing their investments and repaying loans since they no longer have easy access to bank loans. From this emanates the optic of analyzing the impact and the relationship between the size of farmland and the agricultural sustainability of these farms. For this we have chosen an agricultural study area that presents these problems of fragmentation and increased urbanization, which reduces the size of farmland; it is Mornag region.

\section{Materials and methods}

The study area is Mornag region (called in Arabic Mornag Imedat), part of 14 other regions of the delegation with the same name (Mornag). 
The Mornag delegation is located in the governorate of Ben Arous, in the north of Tunisia, at $10 \mathrm{~km}$ from the capital 'Tunis'. The climate is Mediterranean and the dominant crops are fruit trees $(65 \%)$ and cereals (28\%) (Ministry of Environment and sustainable development of Tunisia, 2011) [3]. Urban agglomerations are increasingly invading agricultural land, reducing the area of agricultural land. The region or Imedat of Mornag covers an area of approximately 714.24 ha and 578 farmers. The sample of interviewed farmers is 29 , which is equivalent to $5 \%$. Indeed, it was difficult to obtain socio-economic and even environmental responses from Tunisian farmers. The lockdown period, due to the Covid-19 pandemic, made access to information even more hard.

The method used in this research is the IDEA method (version 4): "Indicateurs de la durabilité des exploitations agricoles" or Farm sustainability indicators. This method was designed to allow a diagnosis of the sustainability of agricultural holdings from direct surveys of farmers (Vilain et al. 2003) ${ }^{[4]}$. Indeed, it is an educational tool implemented to address and analyze various notions in relation to the concept of sustainability. Its main hypothesis is to be able to assess the overall sustainability of an agricultural system by quantifying all of its different characteristics. By then weighting the information obtained from the survey of farmers in Mornag region, then aggregating it, we obtain a score or an overall performance that more or less accurately reflects a real situation of the sustainability of the farm.

In the first step, the sample of our survey was divided into three groups according to the size of the farm in order to be able to calculate and analyze the means of the scores of the indicators obtained in the different components and scales for each group of farms. The sample of farms surveyed is varied. It includes large farms, with areas greater than or equal to 50 ha, medium-sized farms between 10 and 50 ha and small farms with areas less than 10 ha. This distribution was established under the recommendation of the Regional Commissariat for Agricultural Development of Mornag (under the Ministry of Agriculture, water resources and fisheries of Tunisia) given the intensive agricultural vocation of the area. In fact, most of the farmers in the area are affected by land fragmentation. The analysis of the sample showed that the average area of the agricultural holdings surveyed is 84 ha. Small holdings of less than 10 ha represent $45 \%$ of the sample, holdings with total area between 10 and 50 ha represent $24 \%$ of holdings, and large holdings larger than 50 ha represent $31 \%$. These results confirm that agriculture in the Governorate of Ben Arous is based mainly on small farms.

\section{Results and discussions}

In order to analyze in depth the differences among groups of farms as well as the reasons that led to the differences of farmers' behavior, it is necessary to identify in each scale the components and indicators influencing the final result.

\section{Agro-ecological scale}

This scale is divided into three components of equal importance, which contribute to the ecological sustainability of an operation. From the results of the survey and the calculation of the scores for each indicator for the different farms, we were able to quantify the three components of this scale: Diversity, Organization of space, and Agricultural practices rated respectively out of 33; 33 and 34. For a first comparison, we will use the average of the scores assigned to each type of farm in the three components of the agroecological sustainability scale (Figure 1).

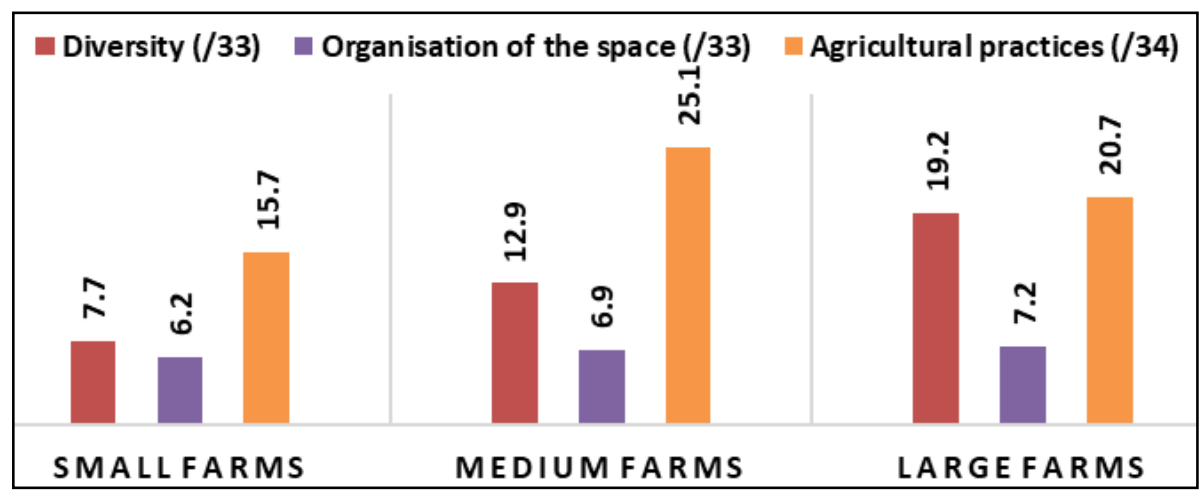

Fig 1: Average of scores for the components of the agroecological scale according to farm size

From this figure, we can see that small farms have the lowest scores in all three components of the agro-ecological scale (diversity, spatial organization, and agricultural practices). Farms of different sizes have averages which are similar in the component 'Organization of space', on the other hand the component 'Diversity' has the best average in the group of large farms (19.2 points) while the component 'Agricultural practices' records the best average in the group of medium-sized farms (25.1 points). In order to be able to better interpret these results, we will analyze the results of the means of the indicators in each component. The scores for each indicator were assigned and calculated based on the results of the surveys and the calculation grid of the IDEA method (version 4).

\section{Diversity component}

The comparison between the averages of the four indicators of the Diversity component for the three groups of farms according to size gave the figure 2 . 


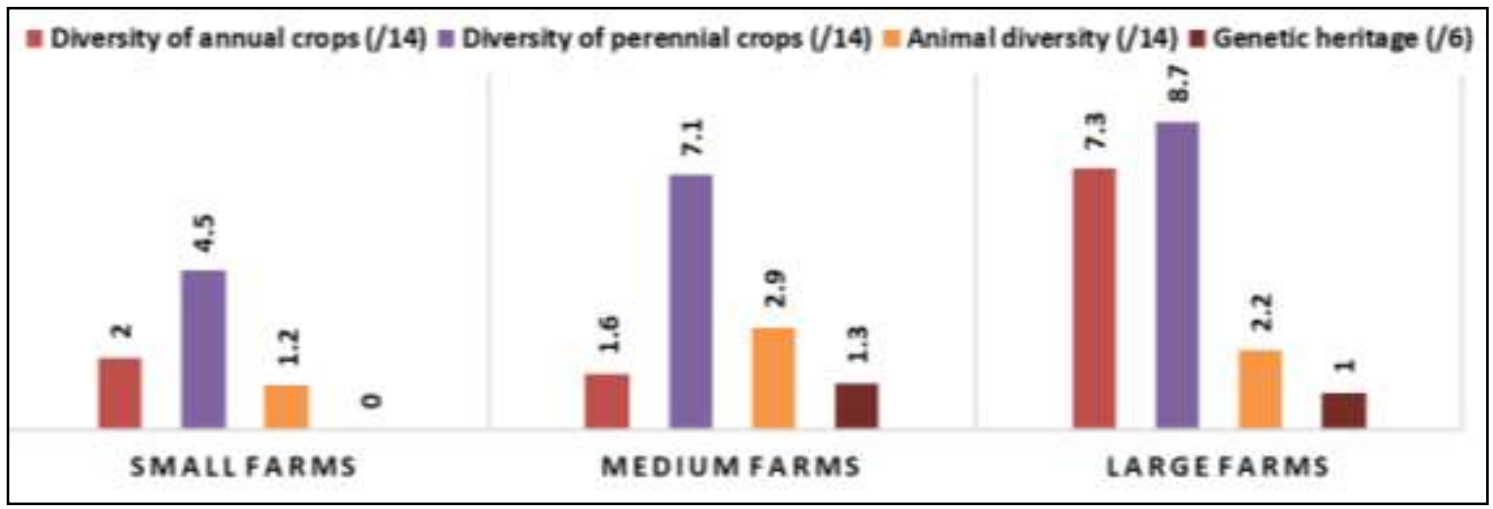

Fig 2: Averages of the scores of the components of the agro-ecological scale according to farm size

From Figure 2, it can be seen that the averages of the component 'Diversity of annual crops' is low for both groups of small and medium-sized farms. On the other hand, it is above the average for the group of large farms. This is explained by the fact that the integration of "field crops" speculation and the integration of different production systems are generally of interest to farmers with large areas. Moreover, in the study area, tree crops are mainly practiced on small and medium-sized farms. On the other hand, the average of the component 'Animal diversity' varies very little between the three groups, which shows that the area of the holding is not an influencing factor. Indeed, even farmers with medium and small areas integrate the practice of breeding in soilless or semi extensive. Finally, the average of the component 'Valorization and conservation of the genetic heritage' is acceptable in the two groups of medium and large farms, which could be explained by the fact that the latter devote part of their plots to cultivate their own plants or some seeds, which is not profitable for smallholders wishing to exploit all the available area.

\section{Organization of space component}

This component has seven indicators that reflect the capacity of the farmer to manage his space well and to use his resources optimally. The comparison between the averages, obtained in the three groups of farms, for the different indicators is shown in the figure 3.

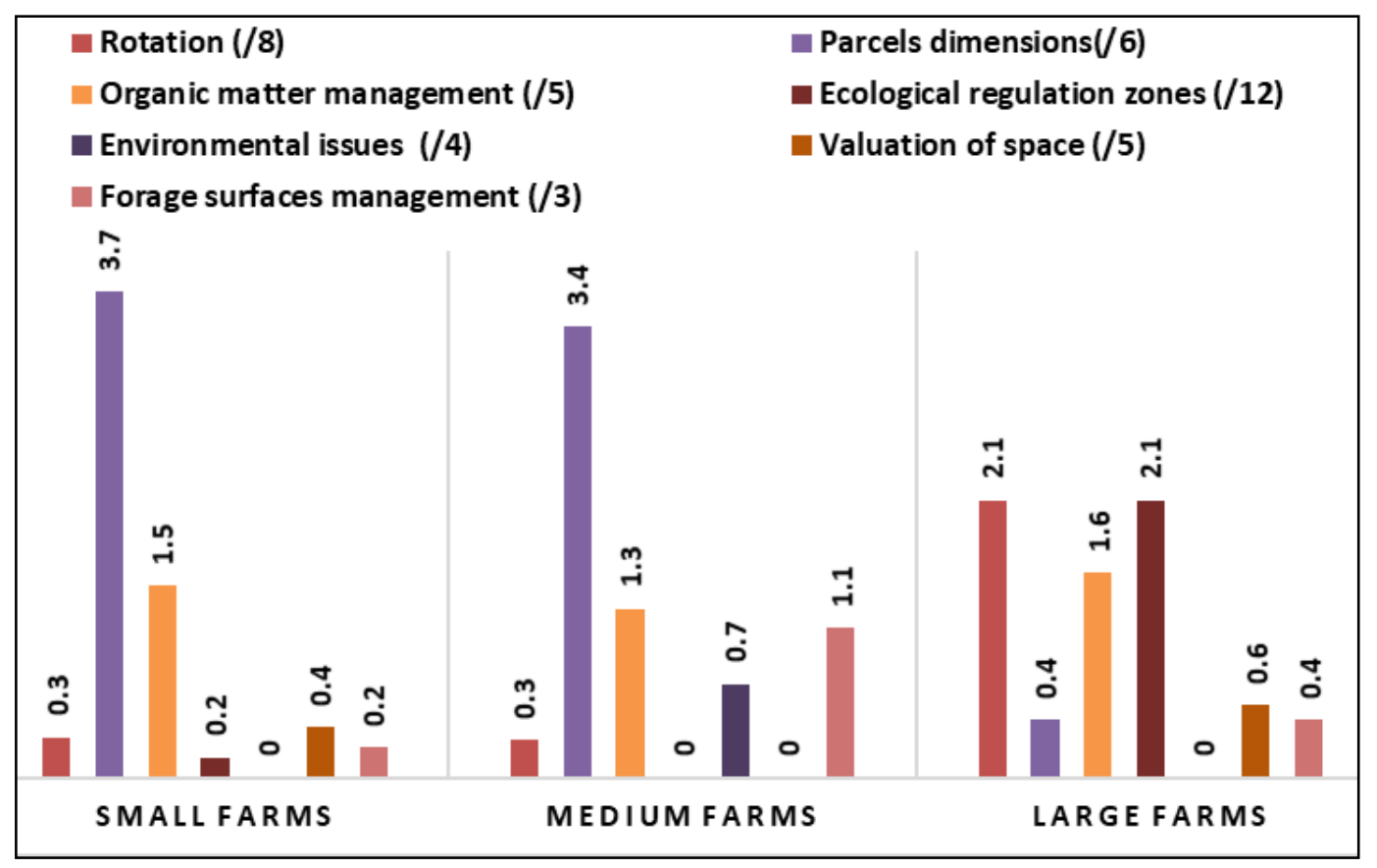

Fig 3: Average scores for the indicators of the Organization of space 'component according to farm size

Since the results obtained for the various indicators of the component "Organization of space", we can see a variability in the means between the three groups. Indeed, the 'Rotation' indicator practically only concerns the group of large farms because these include annual crops, rotation and large crops unlike the two other groups which are mainly arboriculture and monoculture for some. The indicator 'Ecological regulation' concerns all the water and soil conservation activities as well as the zones of regulation of the environmental balance, and mainly concerns the group of large farms as shown in figure 3 . This could be explained by the type of these works and facilities (benches, stone cords, wetlands, courses, etc.) which require a considerable percentage of the total area of the plot. Indeed, small and medium-sized farms want to use all the space to increase profitability and do not think of considering areas reserved for the natural environment and ecological regulation.

\section{Agricultural practices" component}

This last component of the agro-ecological scale contains seven indicators which take into account the nature of the 
agricultural techniques adopted and the farmer's desire to integrate practices favorable to the environment, and which are: fertilization, treatment of effluents, pesticides and veterinary products, animal welfare, protection of soil resources, management of water resources and energy dependency.

The averages of the different groups of farms are given in the figure 4.

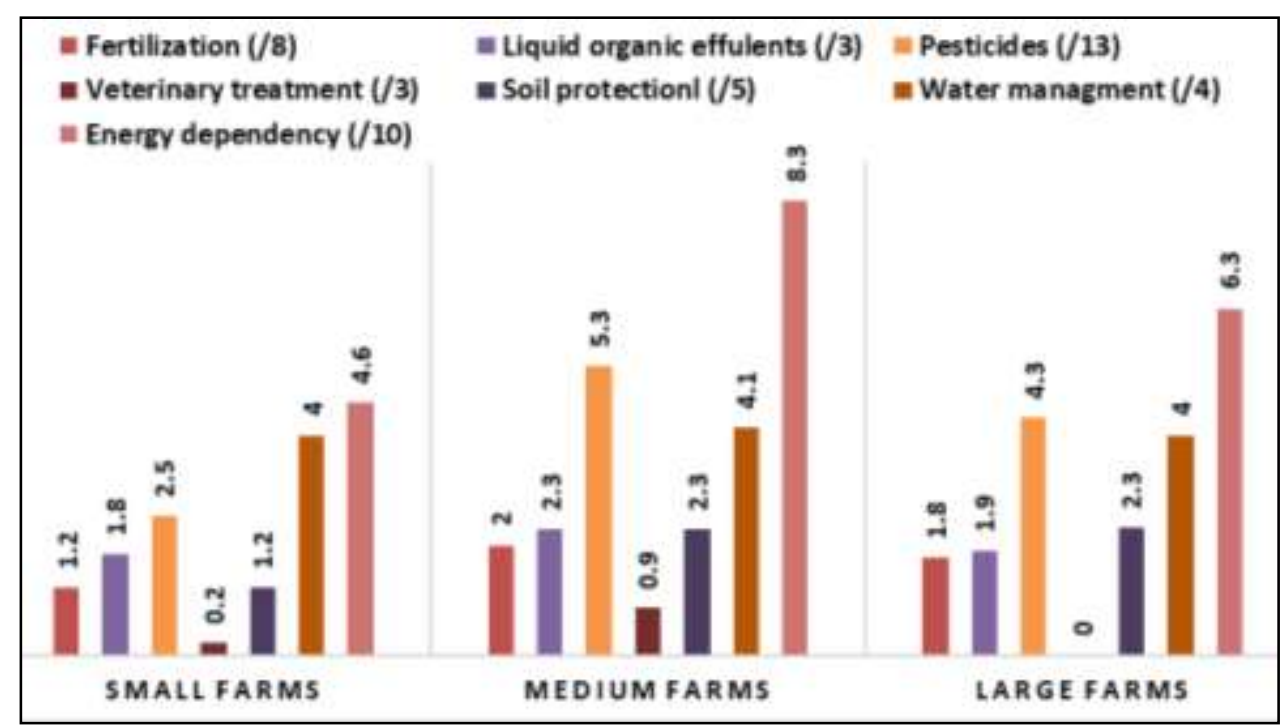

Fig 4: Averages of the indicators of the 'Agricultural practices' component according to farm size

Figure 4 represents the averages of the indicators of the 'Agricultural practices' component in the three groups of farms. It can be seen that the variations between the groups' means are relatively small.

As for the indicator 'Energy dependence' we can see that the means of the second and third group are higher than that of the first group. Indeed, this indicator is calculated from the consumption of non-renewable energy resources of the operation, namely: fuel oil, nitrogen, electricity and the purchase of concentrated feed for livestock. Reducing energy dependency is an indicator of sustainability because it reflects certain autonomy of the production system as well as a good development of resources. Large and mediumsized farms are less dependent on energy than small farms, which could be explained by the fact that a large area and significant capital (assets) make it possible to invest more in costly equipment to enhance local potentials such as renewable energy sources, heat or rainwater recovery as well as the recovery of organic or non-organic waste. Large farms also tend to invest in advanced technical routes and in favor of reducing energy consumption and optimizing inputs such as drip equipment, micro sprinklers, remote management programs and other optimization technologies. This is why they seem to obtain the best averages for this indicator, unlike small farms which are not always able to support such heavy investments and are satisfied with simple or traditional techniques.

The indicator 'Water resource management' underlines the importance of good water management to preserve the longterm potential of soils. It integrates irrigation techniques and devices. This indicator has the best high and close averages in the three groups, which demonstrates that the farmland size factor does not influence the management of the resource. In fact, all the surveyed irrigated farms use the drip system, which helps saving water. For the "Pesticides" indicator, the averages are variable in the three groups; they are higher in the second and third group of farms (medium and large farms). This variation could be explained by the nature of the speculations which differ according to the total area. Indeed, large farms which have diversified crops and integrate large crops and annual crops are not very demanding in pesticides and will have a developed treated area (number of times treated per hectare) less than that of small farms whose total area is often occupied by permanent crops and arboriculture, demanding in treatments.

\section{Socio-territorial scale}

The socio-territorial scale describes the degree of integration of the farm and the farmer in the territory and in society. The analysis of the results obtained by the indicators of this scale will allow assessing the quality of life of the farmer as well as the importance of the market or non-market services that he provides to his territory and to society. Therefore, it provides an idea about the factors and issues beyond just farming. It is divided into three components; each is integrating indicators that reflect the qualitative and quantitative practices of the farmer. The comparison of the means of these components obtained for the three groups of farms of different sizes is presented in the figure below. 


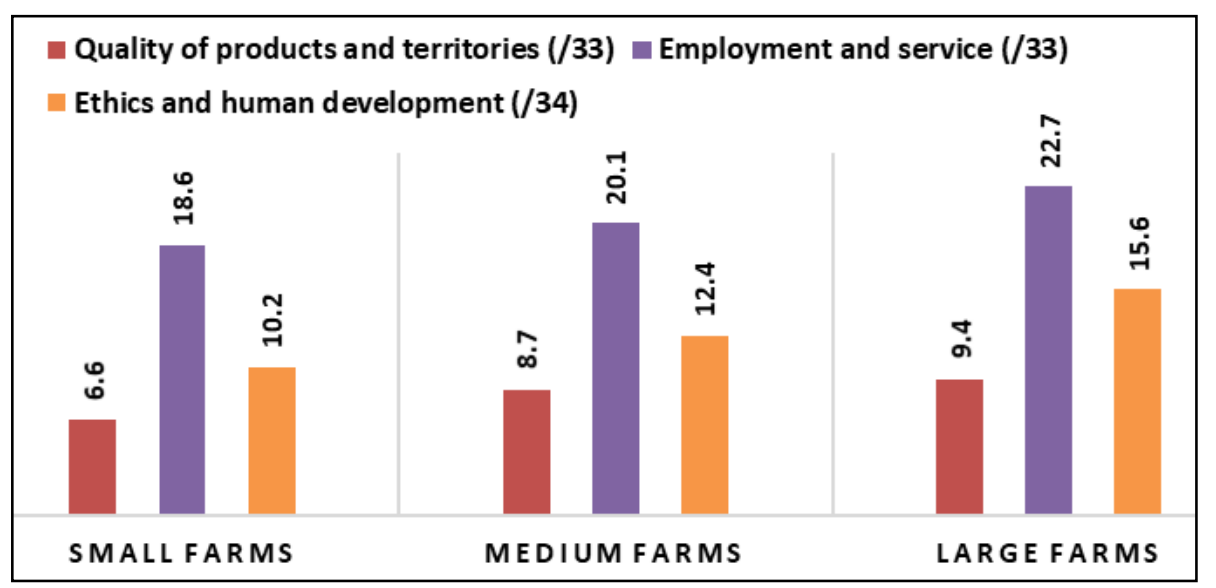

Fig 5: Averages of the scores of the components of the socio-territorial scale according to farm size

From Figure 5, we can see that the averages of the three components increase with the total area of the farm. Nevertheless, they remain low for the three groups, especially in the component 'Product and territory quality'. In order to know the origin of these results and be able to explain them, we will analyze the results for each component and its indicators.

\section{"Product and terroir quality" component}

This first component of the socio-territorial scale seeks to assess the farm in terms of product quality and rural space as well as social integration. It includes five indicators that reflect the impact of the agricultural model adopted on the natural, but also cultural and social environment.

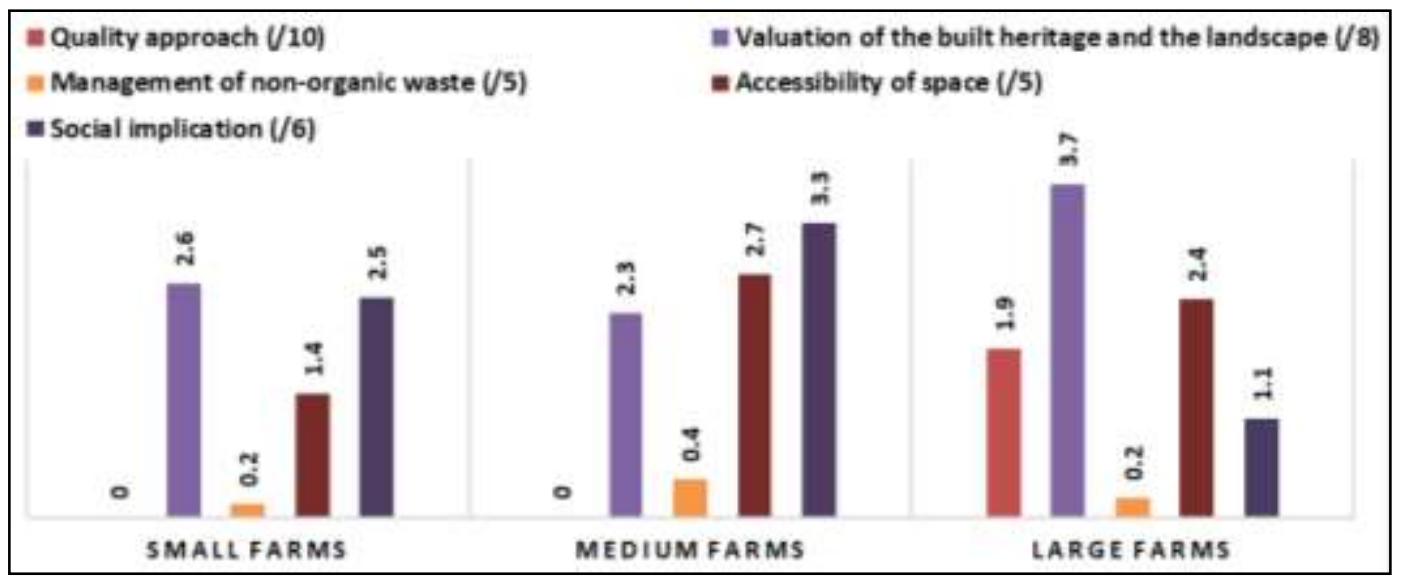

Fig 6: Averages of the indicators of the component "Product quality and terroir" according to farm size

According to Figure 6, the first indicator ('Quality approach') concerns only large farms even though the group's average remains very low. This is mainly due to the importance of the technical and financial means required to be officially recognized by labels or to obtain accreditations by international standards. Indeed, these labels require a technical commitment to adopt a very precise mode of production which is dictated by the specifications. With regard to the valuation of the built heritage and the landscape, the averages of the three groups are slightly closer with a better score for large farms. The latter are therefore more sensitive to the preservation of local customs and the territorial identity of rural areas. In addition, they invest in the maintenance of buildings and infrastructure, improvement of the surroundings and landscaping. This indicator aims to sensitize farmers to the importance of regional identity and working environment and part of its rating is based on self-assessment.

All production activity is concerned by the 'Management of non-organic waste' indicator since it produces waste that must either be recovered or disposed of in a responsible and organized manner. From figure 6, we can see that the means of the three groups are very low. This reflects an almost non-existent level of ecological awareness in Mornag region regardless of the size of the farm. Most of the surveyed farmers do not have any mechanism for valuing or recycling natural resources such as rainwater, they do not sort their waste and in addition practice the burning of waste which is a penalizing action in this indicator given the harmful effects it generates on the environment. It is therefore necessary to make farmers in this area aware of the protection of landscapes and non-renewable resources in order to be able to move towards clean and responsible agriculture. Survey results show that medium and small farms have the best scores for the 'Social involvement' indicator. We have found that smallholders are more interested in participating in non-professional farmers' groups or organizations and structures, which positively affects local dynamics and development. They are also favored in this indicator by their dwelling on or near the farm, since the small plots are most often the result of an inheritance and a family property.

\section{"Employment and services" component}

Large farms stand out from small and medium-sized farms in the 'Autonomy and development of local resources' and 
'Services and multiple activities' indicators. They reflect actions in favor of autonomy and good management of resources, such as: rainwater harvesting, seed autonomy, use of renewable energies, autonomy in fertilizers and animal feed (Figure 7).

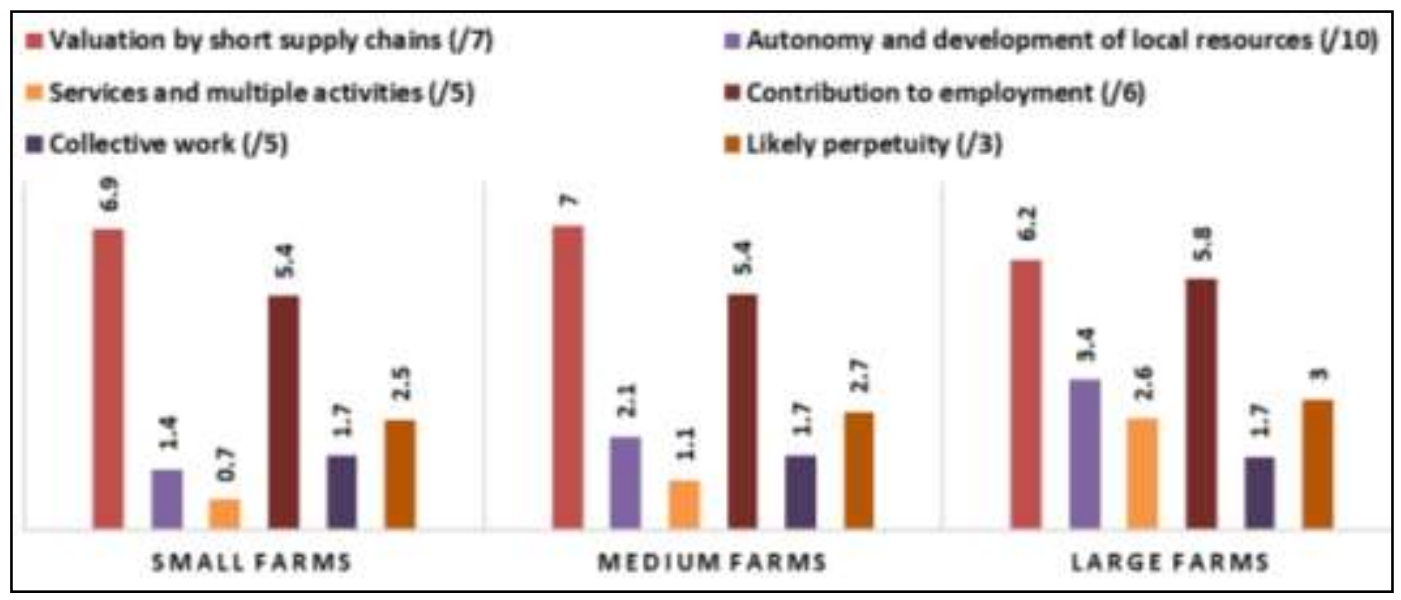

Fig 7: Averages of the indicators of the Employment and services component according to farm size

Likewise, the indicator 'Services and multiple activities' has the best averages in the group of large farms which are characterized by the multifunctionality of agricultural activity and which offer market services for the territory and its inhabitants. Indeed, a large area and significant capital are assets for diversifying services and developing a plurality of products within the same production process. During our investigation, we noticed that this multifunctionality mainly concerned services such as agritourism, the collection and composting of green waste as well as the production of plants or seeds that benefit the inhabitants of the territory. The averages of the other indicators are almost alike in the three groups, showing that the size of the farm is not a factor that significantly influences them.

\section{"Ethics and human development" component}

The "Ethics and Human Development" component includes seven indicators that reflect the importance of the moral and ethical responsibilities of the farmer, going beyond mere economic purposes. Indeed, ethical principles are all the more important as the industrial progress of certain productions has major influences on the ecosystem and its sustainability.

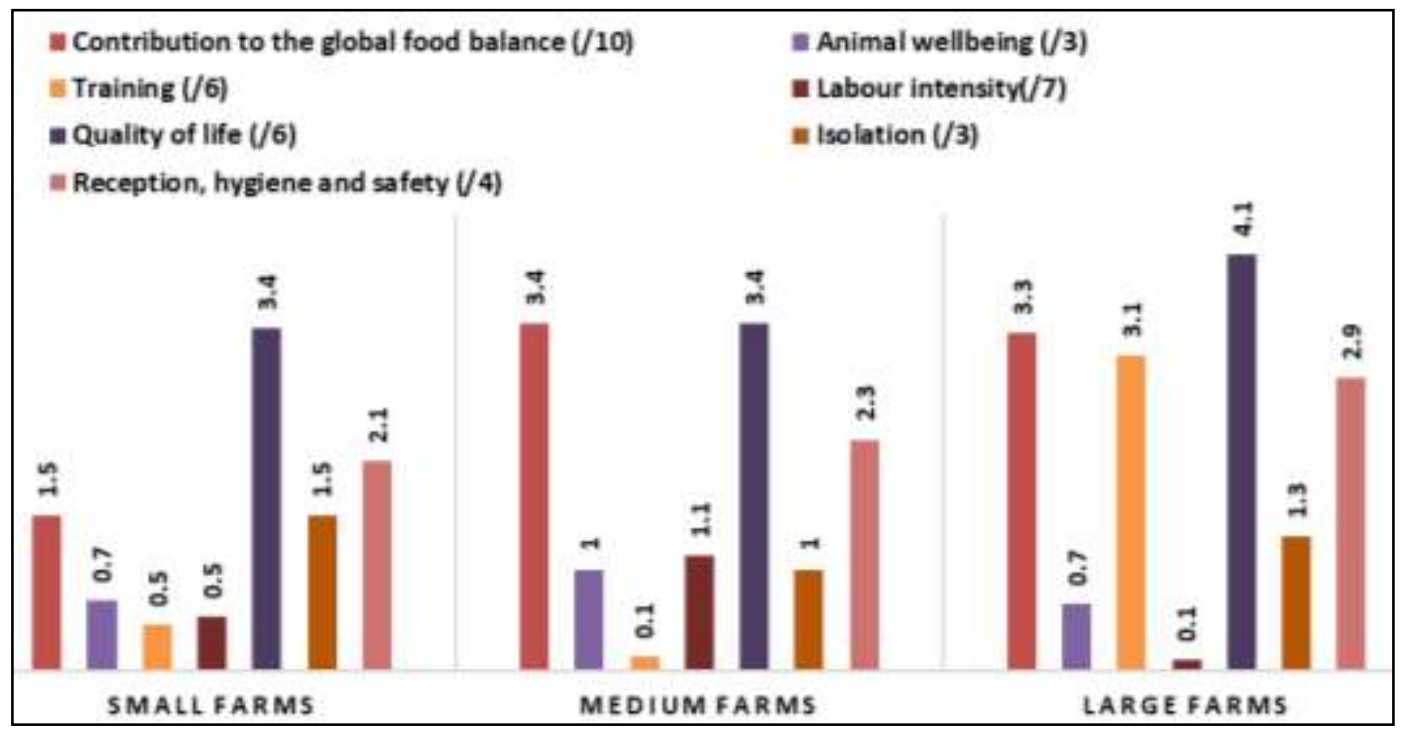

Fig 8: Averages of the indicators of the "Ethics and human development" component according to farm size

The analysis of figure 8 shows us that the indicator 'Contribution to the global food balance mainly concerns medium and large farms. Indeed, it promotes farms with a breeding activity with a low rate of dependence on livestock feed and import of concentrate. Although it records fairly low averages for the three groups, they are a little better on medium and large farms which more often integrate the animal husbandry activity by devoting areas intended for grazing or the production of protein plants and animal feed. Farmers in the study area are therefore quite dependent on imported concentrated feeds and do not devote enough land intended for self-consumption. The third indicator emphasizes that agricultural training is a pledge of the development of the farm and the well-being of the staff. Whether by participating in training courses or by hosting interns or groups of professionals, farmers need to acquire skills and information, to exchange ideas and to meet other actors in order to flourish and evolve. However, we can see that only the large farms in our sample get involved in this kind of activity, maybe for financial reasons or because they 
have more ambitions to expand their activities and improve technical profitability and the development of their teams.

In the 'Work intensity' indicator, which is estimated in relation to the periods of overworked workers per year (number of weeks per year), there are low averages in the three groups and especially that of large farms. This result could be explained by the diversity of activities on these farms which generates multiple harvest periods and therefore an intense seasonal work rate distributed throughout the year. Small plots, on the other hand, tend to have simple production or monoculture, which makes the periods of work overload targeted and less important. Finally, the last three indicators: 'Quality of life', 'Isolation', 'Reception and hygiene' have averages which are similar in the three groups of farms. They include a selfassessment of the farmer in relation to qualitative parameters that combine the professional and personal sphere such as: his satisfaction with the quality of life, his feeling of isolation, and the quality of accommodation of the hand temporary work. We have noticed that these indicators do not depend on the size of the farm but rather on the perception of the farmer.

\section{Economic scale}

A sustainable agricultural operation is based on three poles of sustainability of equal importance. Economic sustainability is therefore a basic condition for sustainable development, and its scale contains four components and six indicators. It assesses the profitability of agricultural production systems not only by their internal efficiency but also by their dependency on the economic and financial support provided by society.

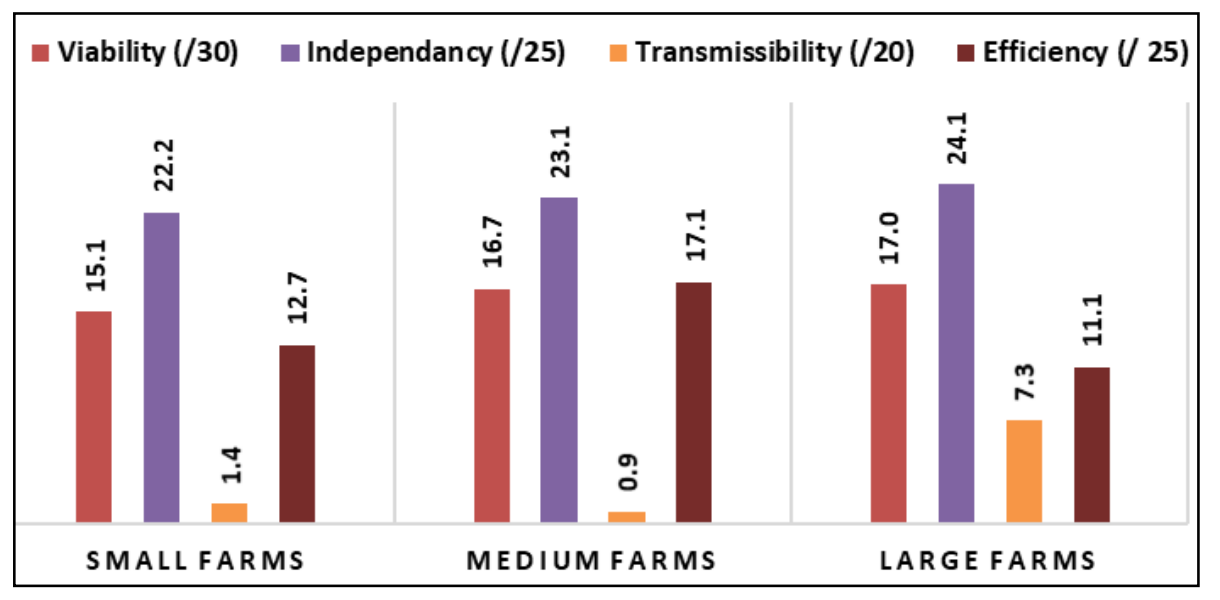

Fig 9: Averages of the scores of the components of the economic scale according to farm size

The analysis of figure 9 shows that the means of the four components vary significantly between the three groups of farms. Indeed, the two components "Economic viability" and "Independency" have close averages in the three groups. On the other hand, the component "Transmissibility" records very low averages with an improvement for the last group and that of Efficiency registers acceptable averages, the best of which is in the second group. In order to better explain the causes of these differences between the three groups of farms, we will then analyze the indicator scores for each component.

\section{Sustainability component}

This component contains two indicators that reflect the economic efficiency of agricultural systems which is a basic condition for the viability and sustainability of the farm.

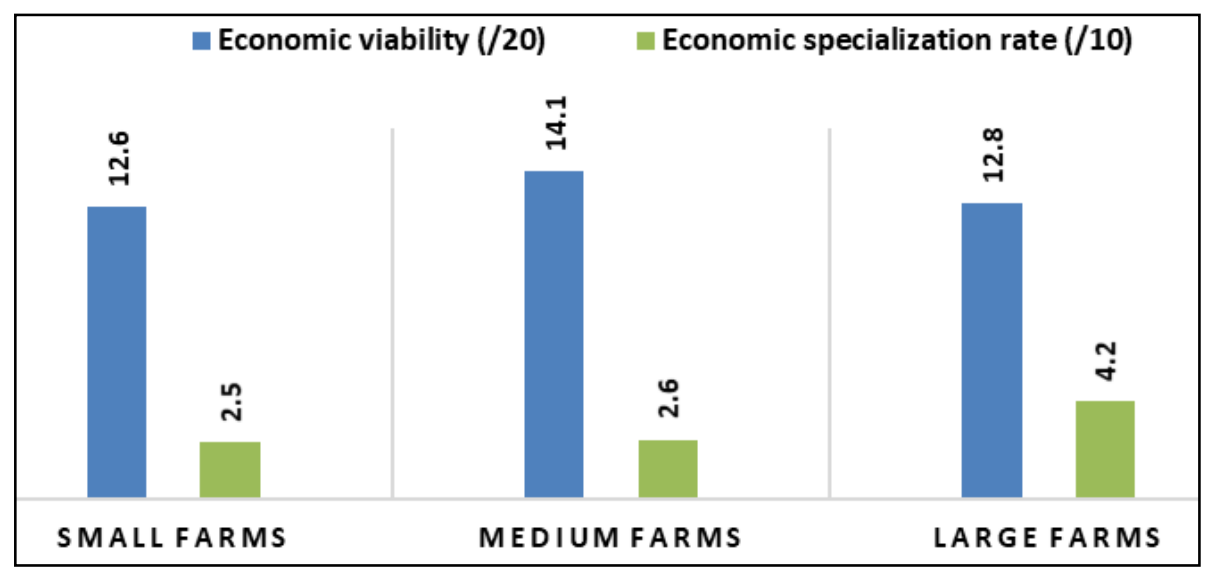

Fig 10: Averages of the indicators of the 'economic viability' component according to farm size

The first indicator concerns the short and medium term economic viability of farms. This is the result of deducting the financing requirements (FR) from the gross operating surplus (GOS), relative to the number of self-employed manpower units (family labor). According to figure 10, the average scores of the indicator are almost equal in the three groups of farms and are acceptable, which is explained by the fact that the number of non-wage labor is low or that GOS is high or even FR is low or neglected. 
Moreover, the speciation rate is low for the three groups of farms. This indicator values diversified production systems and therefore less vulnerable to climatic or economic unforeseen events, but penalizes monoculture and little diversified systems. The low averages recorded are therefore due to the fact that the farmers surveyed do not diversify their productions enough, which makes them more vulnerable to risks. Indeed, the Mornag area is essentially arboricultural and most of the small and medium-sized farms practice simple production systems or even monocultures such as fruit trees, vines, olive trees, etc. In addition, we have noticed that farmers do not diversify their customers either, since for some more than $50 \%$ of the overall turnover is granted by a single customer. This makes the operation extremely vulnerable to economic and market fluctuations.

\section{"Economic and financial independence" component}

It is a component that makes it possible to assess the degree of adaptation of production systems to changes in funding and aid. The question is whether the operation is financially self-sufficient.

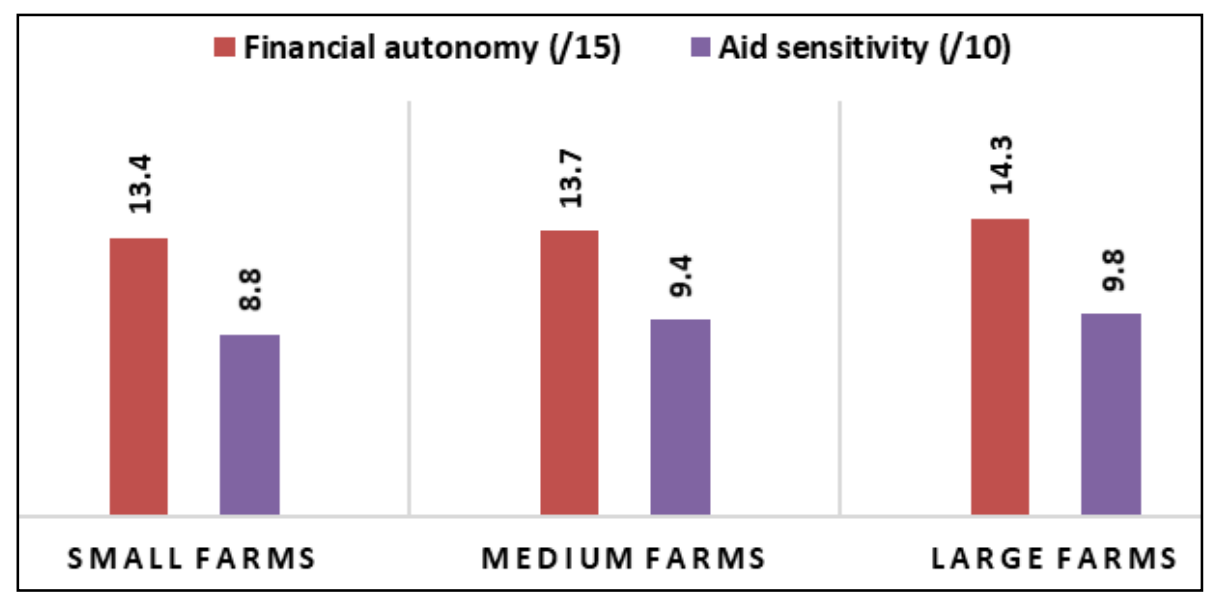

Fig 11: Averages of the indicators of the 'Independency' component according to farm size

We were able to observe that the farmers surveyed have an acceptable margin of maneuver in the face of economic vagaries and loans. Indeed, several farmers told us they were reluctant to bank loans for religious convictions. Likewise, these farmers are not very sensitive to aid (subsidy) and prefer relative autonomy in the method of financing their projects. They are reluctant with regard to public aid and investment support organizations and prefer to rely on their own financial means because of the complexity of the files to be submitted, the deadlines for releasing funds which are too long and the numerous organizations and institutions involved to which they have to make numerous compulsory trips. In addition, many farmers have told us that they no longer have confidence in these institutions and that they believe that the subsidies are not large enough or that their cases have a good chance of not succeeding. . Some are still waiting and others have given up.

\section{"Transmissibility" component}

It is a component that analyzes the capacity of an operation to last over time. It includes a single indicator that reflects the operation of an operation in the event of the manager's cessation of activity or his departure.

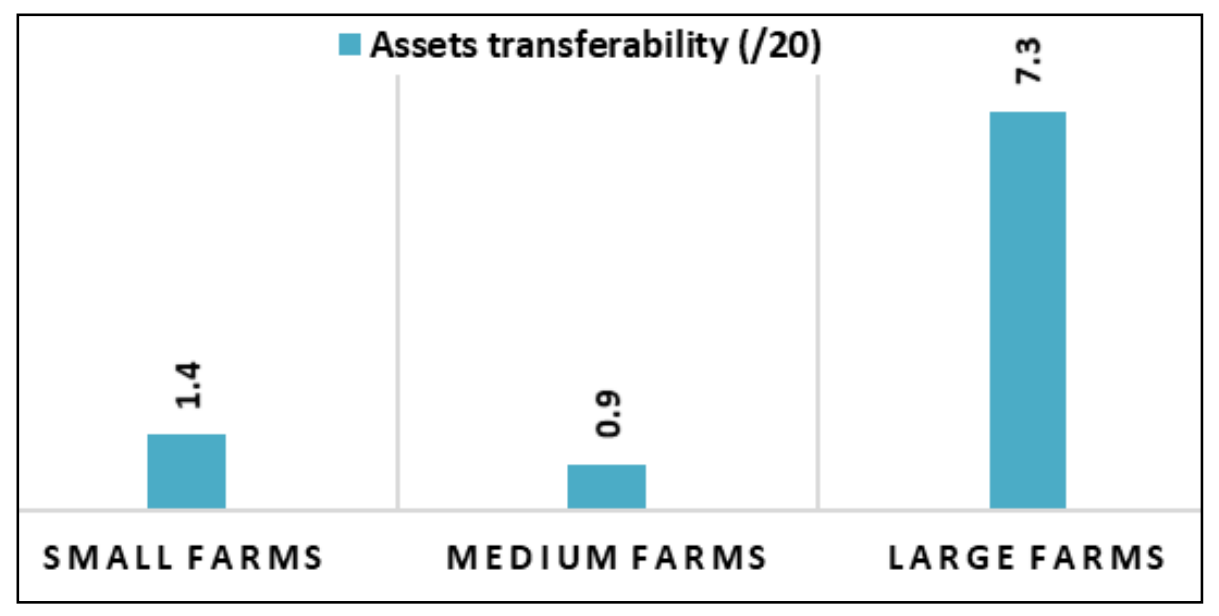

Fig 12: Averages of the indicators of the component "Transmissibility" according to farm size

From the analyzes of the results, we can see that the means of the three groups are low in this indicator. However, the group of large farms records a higher average because they are generally big firms enterprises managed by several partners given the large capital, which improves the succession and therefore the sustainability of the farm through generations. On the other hand, small and mediumsized farms, which have the lowest averages, are characterized by the dominance of small plots owned by one or a few heir farmers, resulting in low transferability of 
capital, especially in the absence of partners. In fact, most of the surveyed small and medium-sized farms are managed only by the household head himself, the children not being involved in the management of the farm. The disinterest of the children and the absence of associates represent a risk for the viability of the exploitation.

\section{"Efficiency of the production process" component}

It contains a single indicator which makes it possible to assess the technical efficiency with which the inputs are managed and processed. It promotes autonomous farms which are able to make the best use of and save their resources.

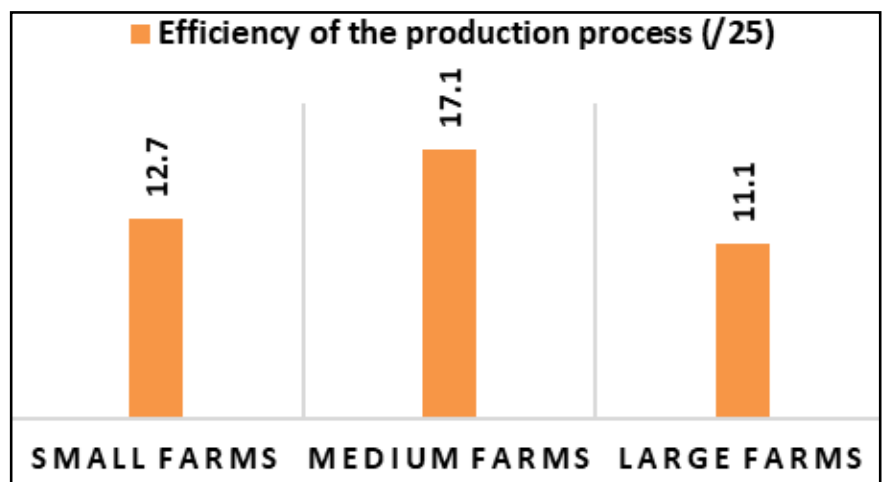

Fig 13: Averages of the indicators of the "Efficiency of the productive process" component according to farm size

Figure 11 shows that the three groups of farms have relatively low averages in this indicator. However, medium farms have a higher average, followed by small and finally large farms. These results could be explained by an imbalance between inputs and revenues from a large operational burden related to processing, marketing or casual labor. This is why the averages of large farms, however profitable and technically advanced, may seem disappointing because of the operational costs of certain production (arboriculture, viticulture, etc.) or heavy wages. In synthesis we can say that each scale is noted on 100 points, and one can notice according to the figure that the average mark is proportional to the area. Indeed, the group of small farms has the lowest scores in the three sustainability scales, followed by the second group and finally the group of large farms with averages slightly higher than the previous one (Figure 12).

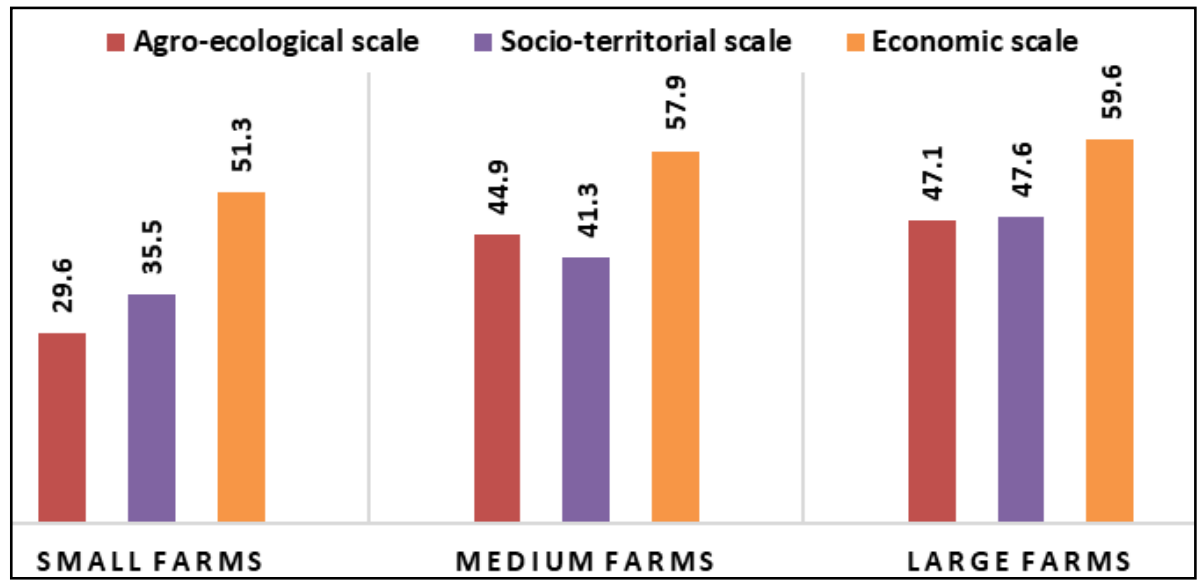

Fig 14: Representation of the means of the three sustainability scales in the three groups of farms according to size

The averages of the agroecological scale vary between 29.6 points and 47.1 points, and correspond to a rather low ecological sustainability for the group of small farms, it is better for the other two groups but although relatively low. In the socio-territorial scale, the group of large farms obtains the best average with 47.6 points while that of small farms has a low average with 35.5 points. With regard to the economic scale, the three averages are quite similar and vary between 51.3 points and 59.6 points. This reflects an average economic sustainability acceptable for the three groups regardless of the size of the farm.

\section{Conclusion}

In conclusion, we can say that at the scale level, the overall scores for each group are positively affected by the total area of the farm. However, detailed analyzes of indicator and component scores have shown us that area is not always a positive factor in sustainability, and in some cases is even a limiting factor. We can therefore note that despite these differences in the averages of the indicators, the overall sustainability of large and medium-sized farms with areas exceeding 10 hectares is better. However, it is useful to take advantage of the failures detected in the various indicators and components to try to improve production systems and facilitate the transition to sustainable agriculture capable of combining economic efficiency and reduction of ecological costs.

\section{References}

1. Grolleau G. Adoption et diffusion des systèmes de management environnemental en agriculture, Séminaire INRA, Paris 2001. 
2. Massin $P$, Kharrat $M$, Farhat $N$, Guenther $N$. Référentiel du développement agricole durable en Tunisie, Document de base pour la formation et le conseil agricole. Agricultural Extension and Training Agency, The Tunisian Ministry of Agriculture, Hydraulic Resources and Fisheries, developed within the framework of the German cooperation PAD project 2016.

3. Ministry of Environment and sustainable development of Tunisia. Direction générale du développement durable. Stratégie Nationale $\mathrm{Du}$ Développement Durable 2011.

4. Vilain L. Indicateurs de durabilité des exploitations agricoles, guide d'utilisation, deuxième édition enrichie et élargie à l'arboriculture, à la viticulture, au maraîchage et à l'horticulture ». Educagri éditions 2003.

5. Zahm F, Ugaglia A, Boureau H, Del'homme B, Barbier JM, Gasselin P, Gafsi M, Guichard L, Loyce C, Manneville V, Menet A, Redlingshofer B. Agriculture et exploitation agricole durables: état de l'art et proposition de définitions revisitées à l'aune des valeurs, des propriétés et des frontières de la durabilité en agriculture. Innovations Agronomiques 2015;46:105-125. 